\title{
Pathogenesis of Venous Thromboembolism: When the Cup Runneth Over
}

\author{
Giuseppe Lippi, M.D., ${ }^{1}$ and Massimo Franchini, M.D. ${ }^{2}$
}

\section{ABSTRACT}

A comprehensive understanding of the pathogenesis of venous thrombosis is essential for identifying patients at increased risk and who may therefore benefit from more aggressive preventive and therapeutic measures. As for other pathologies, the pathogenesis of venous thromboembolism is multifactorial. All risk factors, either congenital or acquired, are relatively "innocent" when considered alone. However, when an individual is unlucky enough to inherit one or more abnormality, compounded in many cases by environmental hazards, that person may be propelled over a threshold that precipitates the development of thrombosis. An appropriate analogy is that where "the last drop makes the cup run over." A reinterpretation of the traditional Virchow's triad (abnormal vessel wall, abnormal blood flow, and abnormal blood constituents) was provided by Eberhard Mammen throughout his research, and this has contributed greatly to the understanding of the pathogenesis of this serious disorder. Mammen postulated immobility as the leading event, because it reduced blood flow as a result of decreased muscle contraction. The subsequent "stasis of flow" led to accumulation of blood within the intramuscular sinuses, especially of the calf, triggering hypercoagulability due to local accumulation of activated clotting factors and coagulation activation products and the simultaneous consumption of blood coagulation inhibitors. On Mammen's "hit list" nearly 20 years ago were included (among inherited abnormalities) decreased protein $\mathrm{C}$, protein $\mathrm{S}$, antithrombin III, plasminogen, and tissue plasminogen activator, and increased plasminogen activator inhibitor-1, whereas (among acquired predisposing conditions) surgery, trauma, previous thromboembolism, prolonged immobility and paralysis, malignancy, congestive heart failure, obesity, advanced age, pregnancy and puerperium, varicose veins, and oral contraceptives were also identified. Some two decades later, the situation has perhaps not changed so much, although studies continue to expand our knowledge of this topic, clarifying the relative contribution of each single risk factor in the pathogenesis of venous thrombosis.

KEYWORDS: Pathogenesis, thrombosis, venous thrombosis, pulmonary embolism, risk factor

Venous thromboembolism (VTE) is a multifaceted clinical entity, which primarily comprises the events of deep vein thrombosis (DVT) and pulmonary embolism (PE). Because specific studies are lacking, the exact prevalence of detectable DVT in patients with acute $\mathrm{PE}$ remains uncertain. An historical meta-analysis
${ }^{1}$ Sezione di Chimica Clinica, Dipartimento di Scienze MorfologicoBiomediche, Università di Verona, Verona, Italy; ${ }^{2}$ Servizio di Immonoematologia e Trasfusione, Azienda Ospedaliero-Universitaria di Parma, Parma, Italy.

Address for correspondence and reprint requests: Prof. Giuseppe Lippi, M.D., Sezione di Chimica Clinica, Dipartimento di Scienze Morfologico-Biomediche, Università degli Studi di Verona, Ospedale Policlinico G.B. Rossi, Piazzale Scuro, 10, 37134 - Verona,
Italy (e-mail: ulippi@tin.it).

A Tribute to Eberhard F. Mammen, M.D. (1930-2008); Guest Editor, Emmanuel J. Favaloro, Ph.D., M.A.I.M.S.

Semin Thromb Hemost 2008;34:747-761. Copyright (C) 2008 by Thieme Medical Publishers, Inc., 333 Seventh Avenue, New York, NY 10001, USA. Tel: +1(212) 584-4662.

DOI 10.1055/s-0029-1145257. ISSN 0094-6176. 
showed that both pathologies are strongly related, as the prevalence of DVT in suspected $\mathrm{PE}$ is $\sim 18 \%$ and in proven $\mathrm{PE}$ (by ventilation/perfusion scintigraphy) is 36 to $45 \%{ }^{1}$ However, a further study employing lower limb venography to detect DVT demonstrated a high prevalence $(82 \%)$ of residual DVT in patients with angiography-proven $\mathrm{PE} .^{2}$ More recently, the same authors observed signs or symptoms of DVT in $32 \%$ of the patients with PE and were able to detect DVT by compression ultrasonography in $60 \%$ of the patients, $45 \%$ having proximal DVT. ${ }^{3}$

The actual incidence and prevalence of VTE is difficult to estimate, due to the silent nature of this disorder and subsequent misdiagnosis. Reliable data, however, indicate an incidence of $\sim 1$ per 1000 annually in adult populations, slightly higher in men than in women, increasing with age and approaching 5 to 6 per 1000 annually by the age of 80 years. ${ }^{4,5}$ The incidence is also heterogeneous among different ethnic groups; a lower rate is reported in the United States, in Asians, Pacific Islanders, and Hispanics compared with that in whites, and some studies have reported an approximate $25 \%$ higher rate in African Americans. ${ }^{4}$
A comprehensive understanding of the pathogenesis of VTE is essential because it would enable the identification of patients at increased risk and who may therefore benefit from reinforced preventive measures and more aggressive therapies in terms of both type and duration. Analogous with other serious and common disorders (e.g., arterial thrombosis, cancer), it is now widely accepted that the pathogenesis of VTE is in fact multifactorial, where any single cause (risk factor) predisposes to but is not sufficient on its own to trigger thrombosis. Consequently, all risk factors, whether congenital or acquired, are relatively "innocent" when considered alone. However, when an individual is unlucky enough to inherit one or more abnormalities, compounded in many cases by environmental hazards, that person may be propelled over a threshold that precipitates the development of thrombosis. An appropriate analogy is that where "the last drop makes the cup run over" (Fig. 1).

The original theory on the pathogenesis of venous thrombosis was formulated by the German physician Rudolf Virchow (1821-1902) in 1856. ${ }^{6}$ Remarkably, this theory (postulated nearly 150 years ago) has lasted

\section{Risk factors for venous thromboembolism}

\section{Congenital}

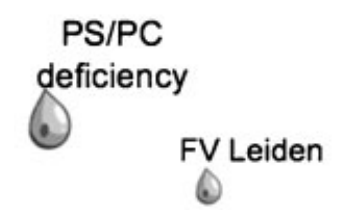

FII (G20210A)

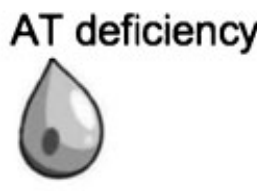

$\mathrm{APCr}$
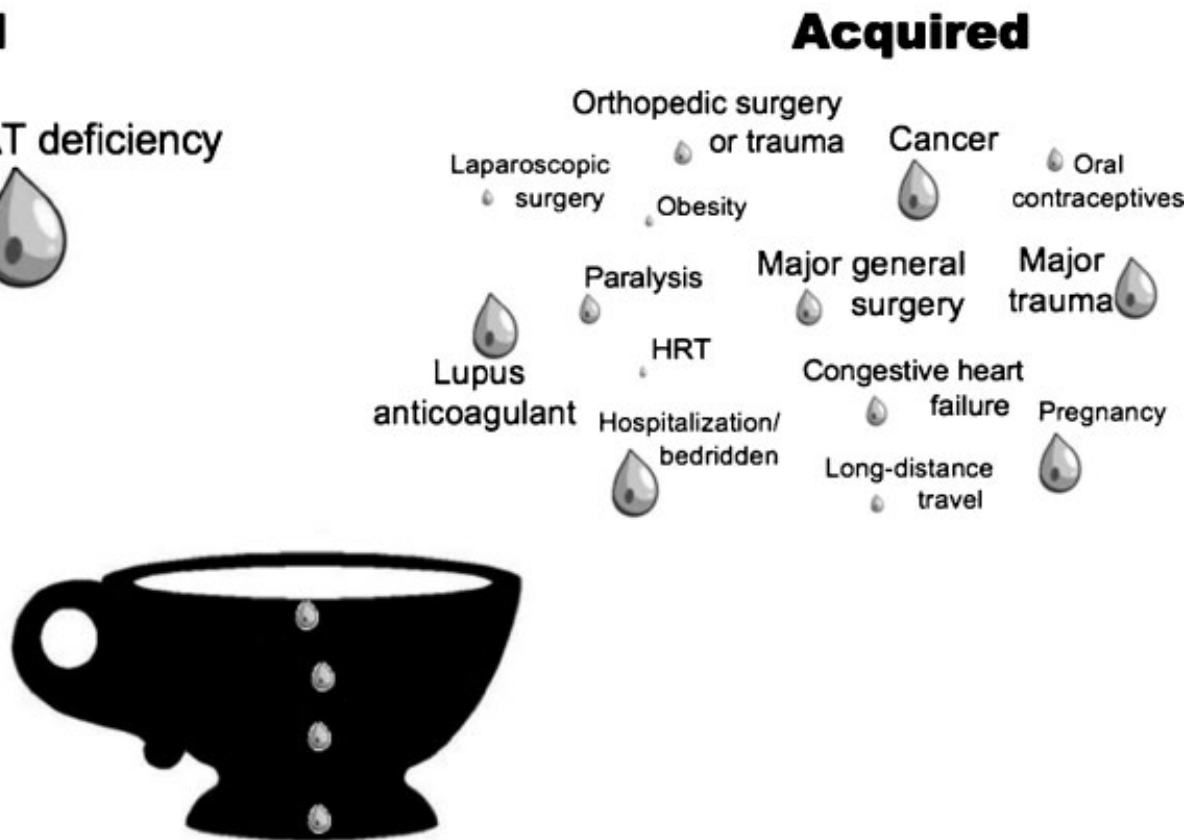

THROMBOSIS

Figure 1 Congenital and acquired risk factors in the pathogenesis of venous thromboembolism. The dimensions of the drops are related to the relative risk of the factors. PC, Protein C; APCr, resistance to activated PC. 
for centuries, and it is still currently considered as relevant. In fact, all the mechanisms involved in the pathogenesis of venous thrombosis can be related to either abnormalities in the endothelium ("abnormal vessel wall"), abnormalities of hemorheology ("abnormal blood flow"), or abnormalities in platelets as well as the coagulation and fibrinolytic pathways ("abnormal blood constituents"). Though all these factors had already been previously established in the medical literature by others, they were eventually referred to as Virchow's triad.

In a seminal review published in 1992, Eberhard Mammen concluded that at least two of these three postulated factors in combination were important in the development of a venous thrombosis, although decreased blood flow or "blood stasis" appeared to be the dominant component. ${ }^{7}$ In fact, though the role of vessel wall damage was uncertain at that time and is still debated now, immobility associated with increased coagulability was considered the leading mechanism. The reinterpretation of Virchow's triad provided by Mammen at that time is even now undisputable: immobility reduces blood flow as a result of decreased muscle contraction. The subsequent accumulation of blood in the intramuscular sinuses, especially of the calf, triggers hypercoagulability due to (a) local accumulation of activated clotting factors and activation products of coagulation and (b) simultaneous consumption of blood coagulation inhibitors. Both mechanisms initiate an autocatalytic process, where activation of coagulation sustains or possibly increases hypercoagulability. Mammen also hypothesized that dilation of the leg veins and reduced venous capacitance might further produce endothelial damage and fibrin nidus formation. ${ }^{7}$ Microscopic vessel wall damage in the onset of venous thrombosis has been observed in patients undergoing hip or knee replacement surgeries and in those with lower limb trauma, burns, and paralysis after spinal cord injury. Conversely, vessel wall damage, either macroscopic or microscopic, has to date not been found in patients subjected to other forms of surgery. ${ }^{7}$

\section{INHERITED THROMBOPHILIA}

Virchow is frequently credited with having first postulated hypercoagulability. However, when the German scientist originally theorized his triad, very little was known about the mechanisms involved in triggering and sustaining blood coagulation, and the proposed "abnormal blood constituents" were only later associated with thrombophilia, a condition that is also referred to as "prothrombotic state" or "hypercoagulable syndromes." ${ }^{4,7}$ When Mammen drafted his seminal review in 1992, patients with any one of the abnormalities he listed (decreased protein C, protein S, antithrombin III, plasminogen, tissue plasminogen activator, or increased plasminogen activator inhibitor-1) were supposed to have an increased risk of VTE. He also emphasized, however, that the presence of a single congenital abnormality was not sufficient to "cause" a thrombosis, a standpoint consistent with the previously mentioned hypothesis of the multifactorial origin of VTE. Over the past 20 years, several other gene variants have been identified as risk factors for VTE (including factor V Leiden and prothrombin G20210A polymorphism).

\section{Antithrombin Deficiency}

Antithrombin is a single-chain glycoprotein belonging to the serine protease inhibitor (serpin) superfamily ${ }^{8}$ and which functions as a natural anticoagulant by binding to, and inactivating, thrombin and the activated coagulation factors IXa, Xa, XIa, and XIIa. ${ }^{9}$ The result of this activity is a reduction in both the generation and the half-life of thrombin. In addition to the active site responsible for coagulation factor inactivation, the antithrombin molecule contains a heparin-binding site. When exogenous heparin or endogenous heparan sulfate binds to this site, the ability of antithrombin to inactivate the above-mentioned factors is greatly enhanced. As expected, any type of mutation that leads to a reduction of antithrombin levels or to a decreased ability to interact with either the activated factors or to heparin will result in an increased risk of thrombosis. ${ }^{10}$ Antithrombin deficiency is mainly transmitted as an autosomal dominant trait, and patients usually present with recurrent VTE episodes during the second to third decade of life. The penetrance of this disease is very high, as most affected family members experience a thrombotic event by the age of 45 years. Antithrombin deficiency is probably the most severe of the inherited thrombophilias, causing upwards of a 20 -fold increased risk for thrombosis compared with that of individuals not carrying this mutation. ${ }^{11}$ However, the prevalence of this mutation in the general population is extremely low, being only $0.02 \%$. Its prevalence among unselected patients with VTE is estimated at 1 to $3 \% .{ }^{12}$ Two major groups of antithrombin deficiencies can be distinguished by laboratory phenotype: in type I deficiency, both antithrombin activity and antigen are reduced to the same extent, whereas in type II deficiency, patients usually exhibit higher antithrombin antigen than activity levels, which is indicative of a functional defect of the antithrombin molecule. ${ }^{13}$ Patients homozygous for type I deficiency have not been described, suggesting that a complete deficiency of antithrombin is incompatible with survival.

\section{Protein C Deficiency}

Eberhard Mammen was recruited from the University of Marburg/Lahn to Wayne State in the late 1950s to work as a research associate in the laboratory of Dr. Walter Seegers. At that time, this laboratory was one of only a 
few investigating coagulation from a biochemical standpoint. Indeed, Dr. Seegers was also the first to isolate clotting factors from plasma, prove their existence, and characterize them. In collaboration, Mammen and Seegers first described protein $\mathrm{C}$, an inhibitor now recognized as one of the major blood clotting inhibitors. ${ }^{14}$ Protein $\mathrm{C}$ is a vitamin $\mathrm{K}$-dependent glycoprotein normally synthesized in the liver. Under physiologic conditions, once activated by the thrombin-thrombomodulin complex, protein $\mathrm{C}$ acts as an anticoagulant by proteolytic degradation of activated coagulant factors $\mathrm{Va}$ and VIIIa. As for other physiologic inhibitors of coagulations, any mutation leading to a reduction of protein $\mathrm{C}$ activity increases the risk of thrombosis. ${ }^{15}$ Inherited protein $\mathrm{C}$ deficiency is transmitted as a dominant autosomal trait. ${ }^{16}$ Patients experience recurrent episodes of VTE, often before the age of 45 years. Homozygous individuals have a more severe clinical picture, not infrequently leading to neonatal purpura fulminans, a potentially fatal condition characterized by multiple thrombosis in small vessels leading to skin necrosis. ${ }^{17}$ The penetrance of the disease is less than that seen in antithrombin deficiency: thus, heterozygous individuals have around a 10-fold increased risk for thrombosis compared with that of the general population. ${ }^{18} \mathrm{How}^{-}$ ever, the disorder is quite rare with a prevalence in the population of only 0.2 to $0.4 \%$ and within 3 to $5 \%$ in patients selected for venous thrombosis. Two types of protein $\mathrm{C}$ deficiency can be recognized. Type I deficiency, which is most common, is characterized by concomitant reduction of protein $\mathrm{C}$ functional and antigen levels due to a reduced synthesis of normal protein $\mathrm{C}$ molecules. In type II deficiency, the functional protein $\mathrm{C}$ level is reduced but the antigen level is normal (typically due to the synthesis of a normal amount of protein $\mathrm{C}$ variant molecules presenting with reduced activity). ${ }^{13}$

\section{Protein S Deficiency}

Protein $\mathrm{S}$ is a vitamin $\mathrm{K}$-dependent protein produced mainly by hepatocytes but also by endothelial cells and megakaryocytes. ${ }^{15}$ It functions as a cofactor of activated protein $\mathrm{C}$ for the degradation of activated factors $\mathrm{Va}$ and VIIIa. Moreover, it is able to directly inactivate factors $\mathrm{Va}$ and $\mathrm{Xa}$. Protein $\mathrm{S}$ circulates in plasma in equilibrium between an inactive form, bound to a carrier called $\mathrm{C} 4 \mathrm{~b}$-binding protein $(\mathrm{C} 4 \mathrm{BP})$, and a free, functionally active form, which accounts for $\sim 40 \%$ of the total plasma protein S. The bioavailability of protein $\mathrm{S}$ is closely linked to the concentration of $\mathrm{C} 4 \mathrm{BP}$, which acts as an important regulatory protein. Thus, all conditions (i.e., pregnancy, oral contraceptive use, acute thrombosis, inflammatory states) associated with increased C4BP levels also cause an increase in bound protein $\mathrm{S}$ and a reduction of the unbound free form. ${ }^{19}$ Transmitted as an autosomal dominant trait, familial protein $\mathrm{S}$ deficiency has a clinical presentation very similar to that observed in protein $\mathrm{C}$ deficiency. ${ }^{20}$ Thus, heterozygous individuals experience early recurrent VTE episodes and sometimes warfarin-induced skin necrosis, and rare homozygotes exhibit a very severe clinical picture with neonatal purpura fulminans. The penetrance of the disease is also similar to that seen in protein $\mathrm{C}$ deficiency, causing a nearly 10 -fold increased risk of venous thrombosis in affected individuals compared with that of the normal population. The prevalence of protein $\mathrm{S}$ deficiency in the general population is estimated at 0.03 to $0.1 \%$, and it occurs in 1 to $3 \%$ of patients with venous thrombosis. ${ }^{19}$ Three types of protein $\mathrm{S}$ deficiency have been defined: type I deficiency is characterized by low total and free protein $S$ antigen and activity, type II deficiency (dysfunctional protein $S$ ) is characterized by normal total and free protein $\mathrm{S}$ antigen but reduced protein $\mathrm{S}$ activity, and type III deficiency is characterized by low free protein $S$ antigen and activity in the presence of normal or subnormal total protein $\mathrm{S}$ antigen level. ${ }^{21}$

\section{Activated Protein C Resistance (Factor V Leiden)}

This concept of resistance to activated protein C (APC) was first described in 1994 by Dahlback and Hildebrand, ${ }^{22}$ who observed that plasma from many patients with venous thrombosis was resistant to the normal anticoagulant effect of APC. In fact, the activated partial thromboplastin time (APTT) of these patients was not prolonged after the addition of APC to their plasma. Successive studies have demonstrated that the great majority of these patients had a mutation in the factor $\mathrm{V}$ gene consisting of a single amino acid change $\left(\mathrm{Arg}^{506}\right.$ to Gln) at one of the APC cleavage sites. ${ }^{23}$ This substitution makes factor $V$ resistant to inactivation by APC. The original mutation identified was called factor $V$ Leiden, as it was initially characterized in Leiden in The Netherlands. Subsequently, other point mutations ( $\mathrm{Arg}^{306}$ to $\mathrm{Thr}$ and $\mathrm{Arg}^{306}$ to Gly) and polymorphisms of factor $\mathrm{V}$ (HR2 haplotype) have been identified, although these are much rarer than factor V Leiden and are without a clear clinical significance. ${ }^{24-26}$ Factor $V$ Leiden is a relatively common mutation, being present in $\sim 5 \%$ of the normal population of northern European origin and in 10 to $50 \%$ of patients presenting with a first episode of VTE. ${ }^{12,27}$ According to the Longitudinal Investigation of Thromboembolism Etiology (LITE) study and the Leiden Thrombophilia (LET) study, patients heterozygous for factor $\mathrm{V}$ Leiden have a relatively low risk for thrombosis, from 3.5 to 8.1 times greater than that of the general population. In contrast, and consistent with the multifactorial view of thrombosis, homozygotes exhibit a very high risk for venous thrombosis, $\sim 24$ to 80 times the normal risk. ${ }^{28,29}$ 


\section{Prothrombin 20210A}

This polymorphism was first described in 1996 by Poort and colleagues, ${ }^{30}$ who showed that a $\mathrm{G}$ to $\mathrm{A}$ transition at nucleotide 20210 of the prothrombin gene within the $3^{\prime}$ untranslated region caused increased basal levels of functionally normal prothrombin, thereby conferring to heterozygous carriers an increased risk (approximately two- to four-fold) for developing venous thrombosis. Thus, these individuals exhibit a relatively low thrombotic risk, and most of them will not have had a thrombotic episode by age 50 years. In contrast, homozygosity for prothrombin gene mutation is much rarer and causes a higher thrombotic risk. Like factor $\mathrm{V}$ Leiden, the prothrombin G20210A polymorphism is quite common, being found in $\sim 2 \%$ of the general population and in 5 to $10 \%$ of selected patients with VTE. ${ }^{31}$ According to the LITE study and the LET study, patients heterozygous for G20210A polymorphism have a relatively low relative risk for thrombosis (1.9 to 2.8 times greater). ${ }^{28,29}$ Remarkably, Margaglione et al hypothesized that the prothrombin G20210A polymorphism might be associated with DVT in the lower extremities alone or when complicated by $\mathrm{PE}$, but not with isolated $\mathrm{PE}{ }^{32}$

\section{Sticky Platelet Syndrome}

The sticky platelet syndrome (SPS) is an autosomal dominant platelet disorder associated with arterial and venous thromboembolic events, characterized by hyperaggregability of platelets in platelet-rich plasma with adenosine diphosphate (ADP) and epinephrine (type I), epinephrine alone (type II), or ADP alone (type III). Clinically, patients may present with angina pectoris, acute myocardial infarction (MI), transient cerebral ischemic attacks, stroke, retinal thrombosis, peripheral arterial thrombosis, and venous thrombosis, frequently recurrent under oral anticoagulant therapy. ${ }^{33}$ Although the role of platelet hyperaggregability as a possible risk factor for VTE is not well defined and this syndrome appears to be prominent especially in patients with unexplained arterial vascular occlusions, combinations of SPS with either congenital thrombophilic defects or acquired disease entities have been described, ${ }^{34}$ and both platelet hyperaggregability and SPS may represent independent risk factors in patients with otherwise unexplained VTE. ${ }^{35}$

\section{ACQUIRED THROMBOPHILIA}

At the time of the seminal review by Mammen in 1992, most acquired risk factors for VTE were already known: surgery and other traumas, previous VTE, prolonged immobility and paralysis, malignancy, congestive heart failure, obesity, advanced age, pregnancy and puerperium, varicose veins, and oral contraceptives $(\mathrm{OCs}){ }^{7}$
Nevertheless, several studies (especially reviews and meta-analyses) contributed over time to further expand the known components of acquired thrombophilia and to elucidate the relative contribution of each single risk factor in the pathogenesis of VTE.

\section{General Surgery}

During extensive tissue damage, the balance between thrombosis and fibrinolysis is altered. Accordingly, Mammen associated three leading thrombogenic factors with surgery and trauma: (1) release of procoagulant material during surgery; (2) preoperative, perioperative, and postoperative immobility; and (3) reduced postoperative fibrinolytic activity (fibrinolytic shutdown). ${ }^{7}$ Before the introduction of routine thromboprophylaxis with heparins, more than 20 years ago, the rates of DVT and fatal PE in both moderate- and high-risk general surgery patients were respectively between $15 \%$ and $30 \%$ and between $0.2 \%$ and $0.9 \% .{ }^{36}$ However, the risk of VTE in contemporary general surgery is uncertain, because studies without thromboprophylaxis are no longer performed. Generally, the risk of VTE varies widely depending on the type of surgery (site, duration, type of anesthesia) and the clinical characteristics of the patients (increasing age, cancer, prior VTE, obesity, comorbidities). Data from the LET study indicate an odds ratio of 5.9 for general surgery. ${ }^{37}$ The risk of DVT in patients undergoing major abdominal surgery, gynecologic surgery, and urologic surgery (in particular open prostatectomy) without thromboprophylaxis is 15 to $30 \% .{ }^{37}$ Colorectal surgery, in particular, carries a specific high risk for postoperative thromboembolic complications compared with that of other types of general surgery, resulting from the frequent presence of several concomitant conditions, including advanced age, pelvic dissection, perioperative positioning of the patients, and presence of cancer or inflammatory bowel disease. ${ }^{38}$ It is also worth mentioning that in the Leiden region, where extended anticoagulant prophylaxis is routinely prescribed for most surgical interventions, $18 \%$ of patients with thrombosis have had a previous surgical intervention, which increased the risk of venous thrombosis sixfold. ${ }^{37}$ Data from other types of surgery are highly heterogeneous.

Since the early 1990s, the demand for cosmetic surgery has grown dramatically in many Western countries, apparently fueled by societal perceptions of the ideal body image. Besides costs, important health care consequences are the potential complications, including thromboembolic disorders, carried by this (mostly unnecessary) type of surgery. In 2001, the American Society of Plastic Surgeons (ASPS) extrapolated existing data to estimate that more than 18,000 cases of DVT may occur in plastic surgery patients each year. Of all common plastic surgery procedures, abdominoplasty has 
the highest rate of thromboembolic complications, with estimates as high as a $1.2 \%$ incidence for DVT and an $0.8 \%$ incidence for PE. ${ }^{39}$ Despite this, more than half of the surgeons responding to an ASPS questionnaire indicated that they currently used no form of thromboprophylaxis. ${ }^{40}$ When liposuction is combined with other procedures, the mortality rate increases from 1 per 47,415 surgeries to 1 per $7314 .{ }^{41}$ Besides surgery and anesthesia, several additional risk factors have been identified in these patients, including smoking, obesity, advanced age, use of hormone replacement or OCs, congestive heart failure, immobilization (bed rests, casts), malignancy, history of previous VTE, and inherited hypercoagulable states, which may act synergistically to increase the individual risk. ${ }^{39}$

\section{Orthopedic Surgery and Trauma}

Major orthopedic surgery involving the lower extremity is a prominent risk factor for VTE, and even with appropriate thromboprophylaxis, total hip or knee replacement will lead to symptomatic VTE in 1 to $3 \%$ of patients. ${ }^{37}$ Globally, the rate of postoperative DVT in patients who do not receive effective prophylaxis is $70 \%$ after nonelective hip surgery and $48 \%$ after elective orthopedic surgery. In a recent analysis of more than 4000 patients, Bannink et al still reported a fourfold increased risk of symptomatic thrombosis after orthopedic surgery. ${ }^{42}$ Perioperative factors affecting risk include venous stasis, direct injury to the veins during surgery, postoperative immobilization, and need for transfusion. Patient-related, or predisposing, risk factors include inherited thrombophilia, advanced age, obesity, varicose veins, and current use of estrogen-containing medications (such as OCs and hormone replacement therapy). Rates of DVT without prophylaxis range from 40 to $60 \%$ seven to fourteen days after major orthopedic surgery; routine ventilation-perfusion scans in patients after hip or knee arthroplasty revealed pulmonary emboli in 3 to $28 \%$ of patients. ${ }^{43}$

Patients who undergo total hip arthroplasty are in the highest risk category for developing postoperative VTE. Evaluation of the natural history of this patient population reveals that the incidence of DVT without appropriate prophylaxis is as high as 32 to $60 \%$, and the incidence of $\mathrm{PE}$ is as high as $16 \%$, with an 0.3 to $3.4 \%$ occurrence of fatal PE. ${ }^{44}$ Patients with hip fractures and without appropriate prophylaxis have a DVT rate of 30 to $60 \%$, with a proximal DVT rate of up to $36 \%$ and a risk of fatal PE from 0.4 to $13 \%$. DVT after knee arthroscopy is a consistent finding in studies of nonprophylaxed patients when routine screening using ultrasound or contrast venography is used. Current data suggest an overall DVT rate of $9.9 \%$ and a proximal DVT rate of $2.1 \%$ after knee arthroscopy without thromboprophylaxis. ${ }^{45}$
Minor injuries of the leg are also associated with greater risk for VTE, as shown in a large, populationbased, case-control study. After adjustment for sex and age, VTE was associated with previous minor injury (adjusted odds ratio $=3.1$ ). The association was strongest for injuries during the 4-week period preceding thrombosis, and no association was evident before 10 weeks. Nevertheless, minor injuries in the leg were more strongly associated with thrombosis (adjusted odds ratio $=5.1$, whereas minor injuries affecting other body parts were not associated with thrombosis. The presence of factor $\mathrm{V}$ Leiden in patients with a leg injury increase the risk up to 50 -fold. ${ }^{46}$

\section{Cancer}

In the challenging cancer biology, transforming genes often act in concert with numerous epigenetic factors, including hypoxia, inflammation, contact between blood and cancer cells, and emission of procoagulant vesicles from tumors, to determine a net imbalance of the hemostatic potential, which is detectable by a variety of laboratory tests. ${ }^{47,48}$ Procoagulant factors in particular are intimately involved in all aspects of hemostatic, cell proliferation, and cellular signaling systems. However, the biggest as-yet unresolved question is why cancer patients develop thrombosis? Because the thrombus itself does not apparently contribute directly to the tumor biology, enhanced hemostasis activation in cancer patients may be interpreted according to the most recent biological evidence. In this perspective, thrombosis may be interpreted as a epiphenomenon of an intricate and effective biological feedback to maintain or promote cancer progression. ${ }^{48}$ In this section, we briefly analyze the pathogenesis and laboratory, clinical, and therapeutic features of cancer and thrombosis.

Trousseau first identified a relationship between thrombosis and malignancy in $1865 .{ }^{49}$ Since then, it has been widely acknowledged that cancer and its related treatments greatly increase the risk of VTE. ${ }^{50}$ Basically, hemostasis and cancer biology interact bidirectionally in a sort of "vicious cycle," where cancer cells are a mighty source of procoagulants (tissue factor, cancer procoagulant, thrombin), which in turn act as strong promoters of cancer growth and spread. ${ }^{48}$ Hence, thromboembolic complications may be the first clinical presentation of cancer; prospective cohort studies documented an increased incidence of cancer after an episode of idiopathic VTE compared with the incidence in the general population. ${ }^{51}$ Moreover, the onset of VTE in patients with cancer is associated with an increased risk of death approximately twofold to eightfold depending on gender and presence or absence of chemotherapy, ${ }^{52,53}$ and patients with cancer who are treated for VTE have higher thrombosis recurrence rates and more hemorrhagic complications compared 
with that of patients without cancer who are treated for VTE. ${ }^{54,55}$

Overall, patients with cancer have a four- to seven-fold increased risk of thrombosis compared with that of the general population. ${ }^{56-59}$ Men are at a greater risk, showing an age-adjusted male-to-female ratio of 1.2:1. ${ }^{53}$ However, data from several epidemiologic investigations demonstrated significant heterogeneity in the risk of VTE according to the different histologic origin. Thodiyil et al, analyzing data from the data set presented in the Medicare Provider Analysis and Review Record (MEDPAR), ${ }^{60}$ calculated the relative risk of VTE by site of malignancy compared with that of subjects with nonmalignant disease and observed greater relative risk for cancer of uterus (3.4), brain (2.4), ovary (2.2), pancreas (2.1), stomach (1.5), kidney (1.4), colon (1.4), lung (1.1), rectum (1.1), and for blood cancers (leukemia 2.2, lymphoma 1.8). The relative risk of other forms of cancer was comparable (prostate, liver, cervix) or even lower (esophagus, breast, bladder, head/neck) than that of patients with various medical conditions who were assumed to have a relative risk of 1.0 for VTE. ${ }^{61}$ In a later article, Heit also estimated the relative risk of VTE for various types of cancer, concluding that pancreatic cancer, lymphoma, and brain cancer have a relative risk for VTE greater than 25, whereas the VTE risk associated with cancer of the ovary, stomach, kidney, colon, rectum, and lung was much lower, albeit still increased $(>17)$ compared with that of persons without cancer. ${ }^{53}$ In the Multiple Environmental and Genetic Assessment (MEGA) study, patients with hematologic malignancies had the highest risk of venous thrombosis (odds ratio $=28$ ), followed by lung cancer and gastrointestinal cancer. The risk of VTE was higher at diagnosis (odds ratio $=54$ ) and in patients with distant metastases (odds ratio $=20){ }^{56}$

Several treatments, including surgery, chemotherapy, and hormone therapy, ${ }^{57}$ along with the placement of a central venous catheter, ${ }^{62}$ also increase the risk of thrombosis. Patients receiving cytotoxic or immunosuppressive chemotherapy have a nearly sixfold increased risk for VTE, probably due to vascular damage induced by several chemotherapeutic agents and release of prothrombotic substances after cell necrosis. ${ }^{63}$ Women who are treated with tamoxifen for breast cancer have a two- to fivefold increased risk of VTE, and the risk is even greater after menopause and when tamoxifen is associated with chemotherapy. ${ }^{64,65}$ Although recently published studies suggest that the incidence of symptomatic and asymptomatic catheter-related thrombosis has decreased significantly in recent years, ${ }^{66}$ cancer patients with a central venous catheter or transvenous pacemaker have a sixfold increase in upper-extremity VTE (odds ratio=6.5). ${ }^{54}$ Finally, the presence of the factor $V$ Leiden mutation in cancer patients determines an increase of nearly 12-fold in the risk versus that of individuals without cancer and factor $\mathrm{V}$ Leiden, similar results being observed in cancer patients carrying the prothrombin 20210 A variant. ${ }^{56}$

\section{Hospital Care Setting}

The rate of VTE, reportedly higher in the hospital than in the ambulatory setting, occurs frequently during and after hospitalization for acute medical illness or surgery due to the higher clot burden and lower rates of VTE prophylaxis. Hence, $\sim 10 \%$ of all deaths in-hospital are still related to $\mathrm{PE}$. In his seminal review of the scientific literature, Mammen provided clear evidence of a relationship between bedrest and venous thrombosis, in that patients confined to bed for more than 1 week have a considerably higher incidence than that of those bedridden for less than 1 week. He also highlighted that preoperative immobility was associated with a higher postoperative incidence of DVT, and postoperative patients remain at higher risk during the entire period of immobility, especially if they remain immobile after discontinuation of thrombosis prophylaxis. ${ }^{7}$ Fifteen years later, Piazza et al strengthened these findings, concluding that hospitalized medical patients had an $\sim 43 \%$ higher relative frequency of $\mathrm{PE}$ compared with that of nonmedical patients. ${ }^{67} \mathrm{PE}$ also accounts for up to $10 \%$ of deaths in hospitalized patients, making VTE the most common preventable cause of in-hospital death. ${ }^{68}$ The Epidemiologic International Day for the Evaluation of Patients at Risk for Venous Thromboembolism in the Acute Hospital Care Setting (ENDORSE) study, a multinational cross-sectional survey designed to assess the prevalence of VTE risk in the acute hospital care setting, estimated that more than half of patients in the hospital care setting (64.4\% surgical and $41.5 \%$ medical) are at risk for VTE according to 2004 American College of Chest Physicians evidence-based consensus guidelines. ${ }^{68}$ Major independent risk factors for VTE in these patients are paresis, acute infectious disease, congestive heart failure, malignancy, an indwelling central venous catheter, varicose veins, and previous thrombosis. Therefore, although the risk varies widely according to the reason for hospitalization and the presence of comorbidities, stroke and infections leading to intensive care management represent conditions at greater risk. In his studies on paralyzed patients after spinal cord injury, Mammen identified a high risk for developing DVT associated with this condition, reporting an overall incidence from 50 to $100 \%$, depending on the methods used for determination, with little relationship to other risk factors. He also reported that the paralyzed limbs in acute stroke patients have a $63 \%$ rate of thrombosis compared with only $7 \%$ in the nonparalyzed. ${ }^{7}$ Highrisk patients also include those hospitalized for chronic inflammatory pathologies, such as lupus erythematosus, 
Wegener granulomatosis, inflammatory bowel diseases, and Behçet's disease. ${ }^{69} \mathrm{~A}$ potential explanation for this finding was again provided by Mammen, who identified abnormality of hemorheology, one of the components of Virchow's triad, as a potential cause. Blood flow slows down as blood viscosity increases; the hyperviscosity syndrome, sustained by dehydration, increased hematocrit values, and increased levels of acute-phase proteins including fibrinogen, is commonplace in patients with inflammatory disorders and in those with spinal cord injury and paralysis. ${ }^{7}$ In patients hospitalized for chronic heart failure, raised venous pressure with subsequent decreased blood flow could be the predisposing factor for the high incidence of VTE. ${ }^{7}$

\section{Immobilization of the Lower Extremities and Long-Distance Travel}

Immobilization is traditionally assumed as a prominent risk factor for thrombosis because it abolishes the essential function of the calf musculature in pumping the blood upstream through the veins. ${ }^{70}$ Mammen originally reported that patients with lower-limbs fractures are at high risk of venous thrombosis, especially when limbs are immobilized by a plastic cast, whereas prophylaxis by external compression modalities greatly reduces the risk, because these devices not only prevent stasis but also apparently activate the fibrinolytic system. ${ }^{7}$ Accordingly, the mean rate of VTE in patients with immobilization of the lower extremities before widespread use of thromboprophylaxis has been reported to be $17 \%$, decreasing to $9.6 \%$ with the use of low-molecular-weight heparin $(\mathrm{LMWH}){ }^{71}$

The association between VTE and air flight was first reported during World War II, where a sixfold increased risk of PE was observed after air raids. ${ }^{72}$ Just 15 years later, Homans designated long-distance travel as a clinical entity, ${ }^{73}$ a condition further translated into the traditional denomination of "economy class syndrome" (combination of immobility and relative dehydration). ${ }^{74}$ Several studies have investigated the relationship between thrombosis and travel over the past decades, but whether long-distance travel and symptomatic VTE are really associated is still a matter of debate, as most travelers who develop DVT or PE also have one or more other predisposing risk factors that include older age, obesity, recent injury or surgery, previous thrombosis, venous insufficiency, malignancy, hormonal therapies, pregnancy, and inherited thrombophilia (especially factor $\mathrm{V}$ Leiden and the prothrombin gene mutation). ${ }^{70} \mathrm{~A}$ recent review has synthesized available data on this topic, concluding that long-distance travel is associated with an increased risk of VTE up to fourfold. The absolute risk of a symptomatic event within 4 weeks of flights longer than 4 hours is 1 in 4600 flights, whereas the risk of acute PE increases with duration of travel, up to 4.8 per million in flights longer than 12 hours. ${ }^{75}$ In a separate article, Ten Wolde et al estimated an accurate odds ratio for the relationship between recent traveling and symptomatic VTE. The analysis consisted of three large case-control studies on patients with clinically suspected DVT and $\mathrm{PE}$. The resulting pooled odds ratio for the association between any medial travel time of 7 hours (quartile range, 4 to 10 hours) and symptomatic VTE was negligible (0.9). When separate analyses were performed for transport by plane, car, bus, train, or boat; no increased risk was identified (odds ratios $=1.2,0.8,0.8,1.4$, and 1.4, respectively). However, a further analysis for duration of travel yielded to an increased odds ratio of 2.5 in the category of 10 to 15 hours of travel. Remarkably, the odds ratio decreased to 1.3 when journeys of more than 15 hours were considered. However, this last finding is probably insignificant, as the study population in the longest duration category was rather small $(n=11){ }^{76}$ Taken together, these data are consistent with the hypothesis that medium- to long-distance travelers have a two- to fourfold increase in relative thrombosis risk compared with that of nontravelers, but the averaged absolute risk is small and there is no evidence that thrombosis is more likely in economy class than in business- or first-class passengers. ${ }^{70}$ Long-distance travelers ( $>10$ hours), conversely, may carry an increased risk of VTE, regardless of the type of transportation.

\section{Oral Contraceptives}

VTE is rare among young women but increases with age. In healthy young women, the estimated incidence of VTE is 1 per 10,000 woman-years of follow-up. Recent estimates by the World Health Organization (WHO) show that more than 100 million women are currently using some form of hormonal contraception worldwide. ${ }^{77}$ The association between female sex hormonecontaining preparations and thrombosis has been documented since the 1960s. ${ }^{78}$ First-generation OCs containing estrogens and high doses of ethinyl estradiol combined with derivatives of norethindrone acetate or nethisterone are no longer available on the market. ${ }^{78}$ Over the past decades, type and dosage of estrogens and progestogens components have been profoundly modified, so that the UK Royal College of Obstetricians and Gynaecologists defines a second-generation combined $\mathrm{OC}$ as a preparation containing 20 to $35 \mu \mathrm{g}$ ethinyl estradiol, with a progestogen (generally norethisterone and levonorgestrel). ${ }^{79}$ The most used third-generation progestogens are gestodene and desogestrel. ${ }^{78}$

A variety of clinical investigations have assessed the risks of thrombosis associated with hormone-based contraceptives. However, due to the variety of preparations and the heterogeneity of the study populations, these studies showed different or even contradictory outcomes. Evidence on OC risk of VTE is mostly derived from 
case-control and nested case-control studies, attributing to $\mathrm{OC}$ use a cumulative risk for thrombosis from 0.89 to 22.1. ${ }^{80}$ OCs use was also associated with 1 to 3 VTE cases per 10,000 woman-years, translating to a two- to sixfold increased relative risk of VTE. ${ }^{80}$ Although thirdgeneration OCs have been reported to increase the risk of VTE compared with that of second-generation OCs, this view is still controversial due to the presence of several confounding variables and study biases. An increased risk of VTE was first reported in the mid-1990s among women using third-generation OCs compared with that of second-generation products, with odds ratios ranging from 1.5 to $2.2 .^{81-84} \mathrm{In}$ a meta-analysis published in the 1999 by Farley et al, third-generation OC use was associated with a significantly increased risk (odds ratio $=1.9$ ) of VTE compared with that of second-generation OCs. ${ }^{85}$ The most recent meta-analysis of Kemmeren et al supported this hypothesis, showing an overall adjusted odds ratio for third- versus secondgeneration of 1.7. Similar risk was found when OCs containing desogestrel or gestodene were compared with those containing levonorgestrel, in both younger and older women. Moreover, the odds ratios for short-term users compared with that of longer-term users were 2.5 and 2.0, respectively. ${ }^{86}$

Although Gomes and Deitcher implicitly confirmed that third-generation OCs might be more thrombogenic, they also concluded that current evidence does not support a recommendation that women already using third-generation OCs stop taking them, given the low absolute risk associated with the use of third-generation OCs for the individual patient. ${ }^{80}$ Overall, this is consistent with an earlier hypothesis by Mammen, who emphasized that there is no convincing evidence that the balance between clotting and fibrinolysis is disturbed in OC users. Accordingly, the risk of thromboembolic complications with use of second- and third-generation OCs is minimal and probably related to underlying congenital or acquired thrombophilic states. ${ }^{87}$ It is worth mentioning, however, that OC use (and pregnancy/ postpartum period) increases the risk of thrombosis in carriers of factor V Leiden to 3.3-fold and 4.2-fold, respectively, whereas other risk factors have a minor effect. ${ }^{88}$ An unequivocal mechanism for explaining the thrombogenicity of OCs (especially estrogen compounds) has not been identified, as several metabolic abnormalities might be triggered to induce a mild prothrombotic state, including increased resistance to APC, increased levels of procoagulant factors VII, IX, X, XII, and XIII, and reduced concentrations of the anticoagulant factors protein $\mathrm{S}$ and antithrombin. ${ }^{37,89}$

Because of the significant risk of VTE in OC users, the 2004 guideline "Venous Thromboembolism and Hormonal Contraception" issued by the UK Royal College of Obstetricians and Gynaecologists recommends that women with current VTE should not use hormonal contraception, whereas women with a personal history of VTE should not use combined oral contraception but may use progestogen-only methods. It is also recommended that combined OC should be discontinued at least 4 weeks before major surgery where immobilization is expected but that progestogen-only methods need not be discontinued prior to surgery even when immobilization is expected and hormonal methods do not need to be discontinued before minor surgery without immobilization. However, it is also suggested that routine thrombophilia screening prior to hormonal contraceptive use is not recommended but may be considered in a woman with a history of VTE in a first-degree relative under the age of 45 years who, after counseling, still wishes to use combined OC. ${ }^{79}$

\section{Pregnancy and Postpartum}

VTE remains a major cause of maternal mortality worldwide, in some geographic areas being ranked as the second most common cause of maternal deaths (rate of maternal deaths from thromboembolism, 0.12 per 10,000 live births and stillbirths)..$^{90}$ In an early study of more than 72,000 deliveries in Scotland, the incidence of DVT was 0.71 per 1000 deliveries with 0.50 occurring in the antenatal period and 0.21 in the puerperium. The incidence of $\mathrm{PE}$ was 0.15 per 1000 deliveries, 0.07 antenatal and 0.08 in the puerperium. ${ }^{91}$ Such a risk of nearly 1 venous thrombotic event per 1000 deliveries is at least a 10-fold increased risk compared with that of nonpregnant women. ${ }^{37}$ Overall, results from studies in which either all or most patients underwent accurate diagnostic testing for VTE report that the incidence of VTE ranges from 0.6 to 1.3 episodes per 1000 deliveries, confirming a 5 -fold to 10 -fold increase in risk compared with that of nonpregnant women of comparable age. ${ }^{92}$ In the most recent MEGA study, however, it has been estimated that the risk of VTE might be nearly 5 -fold increased during pregnancy and up to 60 -fold during the first 3 months after delivery. ${ }^{93}$ A 14 -fold increased risk of deep venous thrombosis of the leg was also observed compared with a 6-fold increased risk of $\mathrm{PE}$, such risk being highest in the third trimester of pregnancy (odds ratio $=8.8)$ and during the first 6 weeks after delivery (odds ratio $=84$ ). Finally, the adjusted relative risk of PE associated with cesarean section compared with that associated with vaginal delivery is $6.7 .^{94}$

The major risk factors for thrombosis during pregnancy include thrombophilia, operative delivery, advanced maternal age, obesity, and preeclampsia; these can be identified in $\sim 70 \%$ of women who develop complications during pregnancy and the puerperium. Thrombophilic abnormalities further affect the risk of thrombosis during pregnancy, particularly antithrombin, factor V Leiden, and prothrombin gene G20210A.90,91 Remarkably, it has been reported that the risk of 
pregnancy-associated VTE might be 11 - to 52-fold increased in factor $\mathrm{V}$ Leiden carriers, 3- to 31-fold increased in carriers of the prothrombin 20210A mutation, and more than 10-fold in those with abnormalities of antithrombin (7-fold for mild deficiency and 64-fold for severe deficiency), protein C (3.6-fold for mild deficiency and 7.2-fold for severe deficiency), or protein S (5-fold for mild deficiency) compared with that of nonpregnant women without these abnormalities. ${ }^{93,95-97}$ Recent studies have also shown an association between thrombophilia and adverse obstetric outcomes such as recurrent miscarriage, preeclampsia, placental abruption, fetal growth retardation, stillbirth, and fetal death. ${ }^{98-100}$

Postpartum DVT is believed related to increased activation of the hemostasis system at the time of delivery. Gerbasi, Mammen, and coauthors investigated 11 hemostatic indices simultaneously in 70 healthy pregnant women, observing a significant increase in fibrinopeptide $A, \beta$-thromboglobulin, and platelet factor 4 , suggesting maximum platelet activation and fibrin formation at the time of delivery. In addition to continued clotting activity at 3 hours postpartum, increased D-dimer, fibrin-fibrinogen degradation products, and decreased $\alpha 2$-antiplasmin levels suggest maximum fibrinolysis. These changes reflected a peak in hemostatic activity at delivery and in the immediate postpartum period that may predispose the development of DVT. ${ }^{101}$

\section{Hormone Replacement Therapy}

Hormone replacement therapy (HRT) is based on administration of sex steroid hormones, primarily estrogens, with or without progesterone. This therapy is widely used for the treatment of menopausal symptoms, to prevent onset of osteoporosis (especially fracture risk), and to lower the burden of cardiovascular events (especially stroke and coronary heart disease). ${ }^{102}$ HRT might also be prescribed to women with premature ovarian failure, which is defined by the occurrence of amenorrhea, hypergonadotropinemia, and estrogen deficiency at a young age ( $<40$ years), and has a prevalence of 0.9 to $1.2 \%$ under the age of 40 years. ${ }^{103}$

Because the dose of estrogens in HRT is much lower than that in OCs, it has been assumed for years that HRT regimens are safer than OCs with respect to the risk of VTE, and the global benefits advocated to HRT in postmenopausal women far outweigh the potential risks. ${ }^{87}$ However, recent evidence attests that HRT is instead associated with an increased risk of stroke, stroke severity, and VTE but not of cardiovascular events. In a recent meta-analysis, including 31 trials (44,113 subjects), HRT was associated with increases in VTE (odds ratio $=2.1$ ), which is comparable with that previously observed for OCs in premenopausal women. Although trials reporting $\mathrm{PE}$ by vital status are too scarce to draw definitive conclusions, ordinal analysis revealed that HRT might more than double the severity of PE. Age has little influence on the risk, whereas the addition of progesterone to estrogens apparently doubles the risk. ${ }^{104}$ Therapy with raloxifene, a selective estrogen receptor modulator indicated for the prevention of osteoporosis in postmenopausal women, is associated with a $62 \%$ increase in odds of either DVT or PE (odds ratio $=1.62$ ), $54 \%$ increase in odds of DVT (odds ratio $=1.54)$, and $91 \%$ increase in odds of $\mathrm{PE}$ alone (odds ratio $=1.91$ ). ${ }^{105}$ Based on available evidence, Gomes and Deitcher concluded that the increased relative risk of VTE among oral HRT users translates into an absolute risk of 2.3 cases per 1000 woman-years, with a two- to four-fold increased relative risk of VTE among oral HRT users compared with that of nonusers. ${ }^{80}$ The most recent meta-analysis of Canonico et al identified a 2.1- to 2.5-fold increased risk for first-time VTE in current users of oral estrogen. ${ }^{106}$ Past users of oral estrogen had a similar risk of VTE to never-users. The risk of VTE in women using oral estrogen was also higher in the first year of treatment (relative risk $=4.0$ ) compared with that of treatment for more than 1 year (relative risk $=2.1$ ).

\section{Obesity}

The association between obesity and thrombotic disorders (especially cardiovascular) is of pivotal importance because obesity is increasing dramatically worldwide. The body mass index (BMI) and the waist-to-hip ratio (WHR) have been considered as risk factors for DVT or correlates of coagulation factors concentrations. Samama reported a twofold increased risk of DVT among outpatients with BMI greater than $30 \mathrm{~kg} / \mathrm{m}^{2} .{ }^{107}$ Such findings were confirmed in a further investigation by White et al, who also observed a relative risk of 2.5 for VTE among patients with a BMI greater than $25 \mathrm{~kg} / \mathrm{m}^{2}$ after hip arthroplasty. ${ }^{108}$ According to a report on 454 consecutive patients with a first episode of objectively diagnosed thrombosis from three anticoagulation clinics in The Netherlands, ${ }^{109}$ obesity $\left(\mathrm{BMI} \geq 30 \mathrm{~kg} / \mathrm{m}^{2}\right)$ increased the risk of thrombosis twofold, adjusted for age and sex. The combined effect of obesity and OCs further increased the risk of thrombosis up to a 10-fold increased risk among younger women ( 15 to 45 years old) with a BMI greater than $25 \mathrm{~kg} / \mathrm{m}^{2}$. Finally, in a recent metaanalysis of 21 case-control and cohort studies with a total of 63,552 patients, the relative risk of obesity for VTE was 2.3. ${ }^{110}$

\section{Antiphospholipid Antibodies}

Antiphospholipid antibodies, such as anticardiolipin antibodies (aCL), are associated with thrombosis and appear to be the most common of the acquired 
blood protein defects. ${ }^{111}$ Patients with systemic lupus erythematosus (SLE) and antiphospholipid antibodies are at a greater risk for VTE than are SLE patients without these antibodies. In a meta-analysis of seven observational studies of risk for antiphospholipidassociated VTE, excluding SLE patients, the overall odds ratio for lupus anticoagulant (LA)-associated VTE was 11. Moreover, the overall odds ratio for aCL-associated VTE was 3.2. ${ }^{112}$ In a systematic review of published articles, Galli and Barbui concluded that LA are a clear risk factor for thrombosis (odds ratio ranging from 4.1 to 16.2), irrespective of the site and type of thrombosis, the presence of SLE, and the methods used to detect them. The aCL and anti- $\beta 2$-glycoprotein I antibodies were also possible risk factors of thrombosis, at least in some selected situations, showing odds ratios from 1.0 to 2.5 and 1.0 to 19.0 , respectively. ${ }^{113}$

\section{Other Risk Factors}

In a recent meta-analysis of 21 case-control and cohort studies with a total of 63,552 patients, the risk of VTE was 1.51 for hypertension, 1.42 for diabetes mellitus, 1.18 for smoking, and 1.16 for hypercholesterolemia, a finding consistent with the hypothesis that cardiovascular risk factors might be associated with VTE, and this association might deserve further scrutiny with respect to individual screening, risk-factor modification, and primary and secondary prevention of VTE. ${ }^{110}$ In a metaanalysis including six case-control studies, incorporating 1826 cases of VTE and 1074 controls, a statistically significant association between lipoprotein(a) levels $>300 \mathrm{mg} / \mathrm{L}$ and VTE (odds ratio $=1.87$ ) was identified. A random-effects model, which accounts for the interstudy variation, yielded a similar estimate of increased risk (odds ratio =1.77). ${ }^{114}$ There is still debate on hyperhomocysteinemia as a risk factor for VTE. A meta-analysis of 10 eligible case-control studies found a pooled estimate of the odds ratio of 2.5 for a fasting plasma homocysteine concentration above the 95 th percentile. For the post-methionine increase in homocysteine concentration, a pooled estimate of 2.6 was found, supporting hyperhomocysteinemia as a mild risk factor for VTE. ${ }^{115}$ More recently, Cattaneo reported that the odds ratio of hyperhomocysteinemia for VTE, as deducted from several prospective studies, might range from 1.2 to $3.4{ }^{116}$

\section{CONCLUSION}

The modern era of the understanding of the etiology of VTE began with Virchow in the mid-1800s. ${ }^{6}$ The main lesson we can learn from the work of Eberhard Mammen on this topic is to regard the pathogenesis of thrombosis within a multifaceted perspective. While reviewing and updating the original theory of Virchow's triad, where all interrelated processes contribute to VTE, he further emphasized that being a risk factor does not necessarily imply that it is the cause of thrombosis, with all factors being important but no single factor being considered essential. ${ }^{7}$ Hence, venous thrombosis often occurs when multiple risk factors, including genetic and environmental, are present at the same time. This is consistent with findings that acquired risk factors (immobilization, surgery and trauma, OC use, pregnancy/postpartum) substantially increase six- to eightfold the risk of thrombosis in carriers of antithrombin, protein $\mathrm{C}$, and protein $\mathrm{S}$ defects. ${ }^{117}$ This image has prompted us to develop the "last drop makes the cup run over" paradigm (Fig. 1). All drops (risk factors) contribute to fill the cup, but none of them, even those that are big, are enough on their own to cause the cup to overflow (i.e., trigger thrombosis). However, when the cup is nearly full, the last drop, even if small, may be sufficient to finally cause the cup to overflow. In such a model, thrombosis is just a probabilistic event; one person may be born with the "cup" empty, someone else with the cup half-full with "congenital drops" (inherited thrombophilia), and some unlucky people are born with the cup nearly full. The "drops of life" (acquired thrombophilia) would ultimately decide the individual's fate in terms of the development of thrombosis.

\section{ABBREVIATIONS}

aCL anticardiolipin antibodies

ADP adenosine diphosphate

APC activated protein $\mathrm{C}$

APTT activated partial thromboplastin time

ASPS American Society of Plastic Surgeons

BMI body mass index

C4BP C4b-binding protein

DVT deep vein thrombosis

HRT hormone replacement therapy

LA lupus anticoagulant

LMWH low-molecular-weight heparin

MEDPAR Medicare Provider Analysis and Review Record

MI myocardial infarction

OC oral contraceptive

$\mathrm{PE}$ pulmonary embolism

SLE systemic lupus erythematosus

SPS sticky platelet syndrome

VTE venous thromboembolism

WHO World Health Organization

WHR waist-to-hip ratio

\section{REFERENCES}

1. van Rossum AB, van Houwelingen HC, Kieft GJ, Pattynama PM. Prevalence of deep vein thrombosis in 
suspected and proven pulmonary embolism: a meta-analysis. Br J Radiol 1998;71:1260-1265

2. Girard P, Musset D, Parent F, Maitre S, Phlippoteau C, Simonneau G. High prevalence of detectable deep venous thrombosis in patients with acute pulmonary embolism. Chest 1999;116:903-908

3. Girard P, Sanchez O, Leroyer C, et al. Evaulation du Scanner Spiralé dans l'Embolie Pulmonaire Study Group. Deep venous thrombosis in patients with acute pulmonary embolism: prevalence, risk factors, and clinical significance. Chest 2005;128:1593-1600

4. Cushman M. Epidemiology and risk factors for venous thrombosis. Semin Hematol 2007;44:62-69

5. Ageno W, Squizzato A, Garcia D, Imberti D. Epidemiology and risk factors of venous thromboembolism. Semin Thromb Hemost 2006;32:651-658

6. Virchow R. Phlogose und Thrombose im Gefäßsystem; Gesammelte Abhandlungen zur Wissenschaftlichen Medizin. Frankfurt, Germany: Staatsdruckerei; 1856

7. Mammen EF. Pathogenesis of venous thrombosis. Chest 1992;102(6, Suppl):640S-644S

8. Spencer F, Becker RC. Diagnosis and management of inherited and acquired thrombophilias. J Thromb Thrombolysis 1999;7:91-104

9. Mammen EF. Antithrombin: its physiological importance and role in DIC. Semin Thromb Hemost 1998;24:19-25

10. Mammen EF. Clinical relevance of antithrombin deficiencies. Semin Hematol 1995;32(4, Suppl 2):2-6

11. Seligsohn U, Lubetsky A. Genetic susceptibility to venous thrombosis. N Engl J Med 2001;344:1222-1231

12. Buchanan GS, Rodgers GM, Branch DW. The inherited thrombophilias: genetics, epidemiology, and laboratory evaluation. Best Pract Res Clin Obstet Gynaecol 2003;17: 397-411

13. Crowther MA, Kelton JG. Congenital thrombophilic states associated with venous thrombosis: a qualitative overview and proposal classification system. Ann Intern Med 2003; 138:128-134

14. Mammen EF, Thomas WR, Seegers WH. Activation of purified prothrombin to autoprothrombin I or autoprothrombin II (platelet cofactor II or autoprothrombin II-A). Thromb Diath Haemorrh 1960;5:218-249

15. Simioni P. The molecular genetics of familial venous thrombosis. Baillieres Best Pract Res Clin Haematol 1999; 12:479-503

16. Griffin JH, Evatt B, Zimmerman TS, et al. Deficiency of protein $\mathrm{C}$ in congenital thrombotic disease. J Clin Invest 1981;68:1370-1373

17. De Stefano V, Finazzi G, Mannucci PM. Inherited thrombophilia: pathogenesis, clinical syndromes and management. Blood 1996;87:3531-3544

18. Franco RF, Reitsma PH. Genetic risk factors of venous thrombosis. Hum Genet 2001;109:369-384

19. Borgel D, Gandrille S, Aiach M. Protein S deficiency. Thromb Haemost 1997;78:351-356

20. Comp PC, Esmon CT. Recurrent venous thromboembolism in patients with a partial deficiency of protein S. N Engl J Med 1984;311:1525-1528

21. Gandrille S, Borgel D, Ireland $\mathrm{H}$, et al. Protein S deficiency: a database of mutations. For the Plasma Coagulation Inhibitors Subcommittee of the Scientific and Standardization Committee of the International Society on Thrombosis and Haemostasis. Thromb Haemost 1997;77: 1201-1214
22. Dahlback B, Hildebrand B. Inherited resistance to activated protein $\mathrm{C}$ is corrected by anticoagulant cofactor activity found to be a property of factor V. Proc Natl Acad Sci U S A 1994;91:1396-1400

23. Bertina RM, Koeleman BP, Koster T, et al. Mutation in blood coagulation factor $\mathrm{V}$ associated with resistance to activated protein C. Nature 1994;369:64-67

24. Williamson D, Brown K, Luddington R, Baglin C, Baglin T, Factor V. Cambridge: a new mutation (Arg306!Thr) associated with resistance to activated protein C. Blood 1998;91:1140-1144

25. Bernardi F, Faioni EM, Castoldi E, et al. A factor V genetic component differing from factor $\mathrm{V}$ R506Q contributes to the activated protein C resistance phenotype. Blood 1997; 90:1552-1557

26. Chan WP, Lee CK, Kwong YL, Lam CK, Liang R. A novel mutation of Arg306 of factor $\mathrm{V}$ gene in Hong Kong Chinese. Blood 1998;91:1135-1139

27. Rees DC, Cox M, Clegg JB. World distribution of factor V Leiden. Lancet 1995;346:1133-1134

28. Rosendaal FR, Koster T, Vandenbroucke JP, Reitsma PH. High risk of thrombosis in patients homozygous for factor $\mathrm{V}$ Leiden. Blood 1995;85:1504-1508

29. Cushman M, Tsai AW, White RH, et al. Deep vein thrombosis and pulmonary embolism in two cohorts: The Longitudinal Investigation of Thromboembolism Etiology. Am J Med 2004;117:19-25

30. Poort SR, Rosendaal FR, Reitsma PH, Bertina RM. A common genetic variation in the $3^{\prime}$-untranslated region of the prothrombin gene is associated with elevated plasma prothrombin levels and an increase in venous thrombosis. Blood 1996;88:3698-3703

31. Rosendaal FR, Doggen CJ, Zivelin A, et al. Geographic distribution of the $20210 \mathrm{G}$ to A prothrombin variant. Thromb Haemost 1998;79:706-708

32. Margaglione M, Brancaccio V, De Lucia D, et al. Inherited thrombophilic risk factors and venous thromboembolism: distinct role in peripheral deep venous thrombosis and pulmonary embolism. Chest 2000;118:1405-1411

33. Mammen EF. Sticky platelet syndrome. Semin Thromb Hemost 1999;25:361-365

34. Frenkel EP, Mammen EF. Sticky platelet syndrome and thrombocythemia. Hematol Oncol Clin North Am 2003;17: 63-83

35. Weber M, Gerdsen F, Gutensohn K, Schoder V, Eifrig B, Hossfeld DK. Enhanced platelet aggregation with TRAP-6 and collagen in platelet aggregometry in patients with venous thromboembolism. Thromb Res 2002;107:325-328

36. Clagett GP, Reisch JS. Prevention of venous thromboembolism in general surgical patients: results of meta-analysis. Ann Surg 1988;208:227-240

37. Rosendaal FR. Venous thrombosis: the role of genes, environment, and behavior. Hematology (Am Soc Hematol Educ Program) 2005:1-12

38. Bergqvist D. Venous thromboembolism: a review of risk and prevention in colorectal surgery patients. Dis Colon Rectum 2006;49:1620-1628

39. Most D, Kozlow J, Heller J, Shermak M. Thromboembolism in plastic surgery. Plast Reconstr Surg 2005;115:20e-30e

40. Rohrich RJ, Rios JL. Venous thromboembolism in cosmetic plastic surgery. Plast Reconstr Surg 2003;112:871-872

41. Hughes CE. Reduction of lipoplasty risks and mortality. Aesth Surg 2001;21:161-163 
42. Bannink L, Doggen CJM, Nelissen RGHH, Rosendaal FR. Increased risk of venous thrombosis after orthopedic and general surgery: results of the MEGA study [abstract]. J Thromb Haemost 2005;3(Suppl 1):1653

43. White RH, Zhou H, Romano PS. Incidence of symptomatic venous thromboembolism after different elective or urgent surgical procedures. Thromb Haemost 2003;90: 446-455

44. Pineo GF, Hull RD. Prophylaxis of venous thromboembolism following orthopaedic surgery: mechanical and pharmacological approaches and the need for extended prophylaxis. Thromb Haemost 1999;82:918-924

45. Ilahi OA, Reddy J, Ahmad I. Deep venous thrombosis after knee arthroscopy: a meta-analysis. Arthroscopy 2005;21: 727-730

46. van Stralen KJ, Rosendaal FR, Doggen CJ. Minor injuries as a risk factor for venous thrombosis. Arch Intern Med 2008;168:21-26

47. Piccioli A, Falanga A, Baccaglini U, Marchetti M, Prandoni P. Cancer and venous thromboembolism. Semin Thromb Hemost 2006;32:694-699

48. Franchini M, Montagnana M, Targher G, Manzato F, Lippi G. Pathogenesis, clinical and laboratory aspects of thrombosis in cancer. J Thromb Thrombolysis 2007;24: 29-38

49. Trousseau A. Phlegmasia alba dolens. In: Clinique Médicale de l'Hôtel-Dieu de Paris. Paris: Ballière; 1865:654-712

50. Bergqvist D. Venous thromboembolism in cancer patients: expanding horizons. Semin Thromb Hemost 2002;28 (Suppl 3):19-23

51. Agnelli G. Venous thromboembolism and cancer: a two way clinical association. Thromb Haemost 1997;78:117-120

52. Sorensen HT, Mellemkjaer L, Olsen JH, Baron JA. Prognosis of cancers associated with venous thromboembolism. N Engl J Med 2000;343:1846-1850

53. Heit JA. Cancer and venous thromboembolism: scope of the problem. Cancer Control 2005;12(Suppl 1):5-10

54. Hutten BA, Prins MH, Gent M, Ginsberg J, Tijssen JG, Buller HR. Incidence of recurrent thromboembolic and bleeding complications among patients with venous thromboembolism in relation to both malignancy and achieved international normalized ratio: a retrospective analysis. J Clin Oncol 2000;18:3078-3083

55. Prandoni P, Lensing AWA, Piccioli A, et al. Recurrent venous thromboembolism and bleeding complications during anticoagulant treatment in patients with cancer and venous thrombosis. Blood 2002;100:3484-3488

56. Blom JW, Doggen CJM, Osanto S, Rosendaal FR. Malignancies, prothrombotic mutations, and the risk of venous thrombosis. JAMA 2005;293:715-722

57. Heit JA, Silverstein MD, Mohr DN, Petterson TM, O'Fallon WM, Melton LJ III. Risk factors for deep vein thrombosis and pulmonary embolism: a population-based case-control study. Arch Intern Med 2000;160:809-815

58. Silverstein MD, Heit JA, Mohr DN, Petterson TM, O'Fallon WM, Melton LJ III. Trends in the incidence of deep vein thrombosis and pulmonary embolism: a 25 -year populationbased study. Arch Intern Med 1998;158:585-593

59. Sutherland DE, Weitz IC, Liebman HA. Thromboembolic complications of cancer: epidemiology, pathogenesis, diagnosis, and treatment. Am J Hematol 2003;72:43-52

60. Levitan N, Dowlati A, Remick SC, et al. Rates of initial and recurrent thromboembolic disease among patients with malignancy versus those without malignancy. Risk analysis using Medicare claims data. Medicine (Baltimore) 1999;78: 285-291

61. Thodiyil PA, Kakkar AK. Variation in relative risk of venous thromboembolism in different cancers. Thromb Haemost 2002;87:1076-1077

62. Lee AY, Levine MN. The thrombophilic state induced by therapeutic agents in the cancer patient. Semin Thromb Hemost 1999;25:137-145

63. Bick RL. Cancer-associated thrombosis. N Engl J Med 2003; 349:109-110

64. Fisher B, Costantino J, Redmond C, et al. A randomized clinical trial evaluating tamoxifen in the treatment of patients with node-negative breast cancer who have estrogenreceptor-positive tumors. N Engl J Med 1989;320:479-484

65. Pritchard KI, Paterson AH, Paul NA, et al. Increased thromboembolic complications with concurrent tamoxifen and chemotherapy in a randomized trial of adjuvant therapy for women with breast cancer. National Cancer Institute of Canada Clinical Trials Group Breast Cancer Site Group. J Clin Oncol 1996;14:2731-2737

66. Freytes CO. Thromboembolic complications related to central venous access catheters in cancer patients. Semin Thromb Hemost 2007;33:389-396

67. Piazza G, Seddighzadeh A, Goldhaber SZ. Double trouble for 2,609 hospitalized medical patients who developed deep vein thrombosis: prophylaxis omitted more often and pulmonary embolism more frequent. Chest 2007;132:554-561

68. Cohen AT, Tapson VF, Bergmann JF, et al. ENDORSE Investigators. Venous thromboembolism risk and prophylaxis in the acute hospital care setting (ENDORSE study): a multinational cross-sectional study. Lancet 2008;371:387394

69. Le Jeune S, Pistorius MA, Planchon B, Pottier P. Risk of venous thromboembolism in acute medical illnesses. Part 2: situations at risk in ambulatory, hospital and internal medicine settings. Rev Med Interne 2008;29:462-475

70. Gallus AS. Travel, venous thromboembolism, and thrombophilia. Semin Thromb Hemost 2005;31:90-96

71. Ettema HB, Kollen BJ, Verheyen CC, Büller HR. Prevention of venous thromboembolism in patients with immobilization of the lower extremities: a meta-analysis of randomized controlled trials. J Thromb Haemost 2008;6: 1093-1098

72. Simpson K. Shelter deaths from pulmonary embolism. Lancet 1940;ii:744

73. Homans J. Thrombosis of the deep leg veins due to prolonged sitting. N Engl J Med 1954;250:148-149

74. Cruickshank JM, Gorlin R. Air travel and thrombotic episodes: the economy class syndrome. Lancet 1988;2: 497-498

75. Kuipers S, Schreijer AJ, Cannegieter SC, Büller HR, Rosendaal FR, Middeldorp S. Travel and venous thrombosis: a systematic review. J Intern Med 2007;262:615-634

76. Ten Wolde M, Kraaijenhagen RA, Schiereck J, et al. Travel and the risk of symptomatic venous thromboembolism. Thromb Haemost 2003;89:499-505

77. United Nations Population Division. World Contraceptive Use 2001. New York, NY: United Nations; 2002

78. Watson HG. Sex hormones and thrombosis. Semin Hematol 2007;44:98-105

79. Royal College of Obstetricians and Gynaecologists (RCOG). Venous Thromboembolism and Hormonal Contraception. 
Guideline no. 40. London, United Kingdom: Royal College of Obstetricians and Gynaecologists; 2004

80. Gomes MP, Deitcher SR. Risk of venous thromboembolic disease associated with hormonal contraceptives and hormone replacement therapy: a clinical review. Arch Intern Med 2004;164:1965-1976

81. World Health Organization Collaborative Study of Cardiovascular Disease and Steroid Hormone Contraception. Venous thromboembolic disease and combined oral contraceptives: results of international multicentre case-control study. Lancet 1995;346:1575-1582

82. Jick H, Jick SS, Gurewich V, Myers MW, Vasilakis C. Risk of idiopathic cardiovascular death and nonfatal venous thromboembolism in women using oral contraceptives with differing progestagen components. Lancet 1995;346: 1589-1593

83. Bloemenkamp KW, Rosendaal FR, Helmerhorst FM, Buller HR, Vandenbroucke JP. Enhancement by factor V Leiden mutation of risk of deep-vein thrombosis associated with oral contraceptives containing a third-generation progestagen. Lancet 1995;346:1593-1596

84. Spitzer WO, Lewis MA, Heinemann LA, Thorogood M, MacRae KD. Third generation oral contraceptives and risk of venous thromboembolic disorders: an international casecontrol study. BMJ 1996;312:83-88

85. Farley TM, Meirik O, Collins J. Cardiovascular disease and combined oral contraceptives: reviewing the evidence and balancing the risks. Hum Reprod Update 1999;5:721-735

86. Kemmeren JM, Algra A, Grobbee DE. Third generation oral contraceptives and risk of venous thrombosis: metaanalysis. BMJ 2001;323:131-134

87. Mammen EF. Oral contraceptive pills and hormonal replacement therapy and thromboembolic disease. Hematol Oncol Clin North Am 2000;14:1045-1059

88. Simioni P, Sanson BJ, Prandoni P, et al. Incidence of venous thromboembolism in families with inherited thrombophilia. Thromb Haemost 1999;81:198-202

89. Lippi G, Manzato F, Brocco G, Franchini M, Guidi G. Prothrombotic effects and clinical implications of thirdgeneration oral contraceptives use. Blood Coagul Fibrinolysis 2002;13:69-72

90. Tan JY. Thrombophilia in pregnancy. Ann Acad Med Singapore 2002;31:328-334

91. McColl MD, Ramsay JE, Tait RC, et al. Risk factors for pregnancy associated venous thromboembolism. Thromb Haemost 1997;78:1183-1188

92. Bates SM, Greer IA, Pabinger I, Sofaer S, Hirsh J. Venous thromboembolism, thrombophilia, antithrombotic therapy, and pregnancy: American College of Chest Physicians Evidence-Based Clinical Practice Guidelines (8th Edition). Chest 2008;133:844S-886S

93. Pomp ER, Lenselink AM, Rosendaal FR, Doggen CJ. Pregnancy, the postpartum period and prothrombotic defects: risk of venous thrombosis in the MEGA study. J Thromb Haemost 2008;6:632-637

94. Ros HS, Lichtenstein P, Bellocco R, et al. Pulmonary embolism and stroke in relation to pregnancy: how can high-risk women be identified? Am J Obstet Gynecol 2002; 186:198-203

95. Martinelli I, De Stefano V, Taioli E, Paciaroni K, Rossi E, Mannucci PM. Inherited thrombophilia and first venous thromboembolism during pregnancy and puerperium. Thromb Haemost 2002;87:791-795
96. Gerhardt A, Scharf RE, Beckmann MW, et al. Prothrombin and factor $\mathrm{V}$ mutations in women with a history of thrombosis during pregnancy and the puerperium. $\mathrm{N}$ Engl J Med 2000;342:374-380

97. Gerhardt A, Scharf RE, Zotz RB. Effect of hemostatic risk factors on the individual probability of thrombosis during pregnancy and the puerperium. Thromb Haemost 2003; 90:77-85

98. Gammaro L, Lippi G, Bottini E, et al. Severe preeclampsia in antithrombin III deficiency with no history of venous thromboembolism. J Nephrol 2001;14:312-315

99. Brenner B. Thrombophilia and fetal loss. Semin Thromb Hemost 2003;29:165-170

100. Rodger MA, Paidas M. Do thrombophilias cause placentamediated pregnancy complications? Semin Thromb Hemost 2007;33:597-603

101. Gerbasi FR, Bottoms S, Farag A, Mammen EF. Changes in hemostasis activity during delivery and the immediate postpartum period. Am J Obstet Gynecol 1990;162: $1158-1163$

102. Hickey M, Davis SR, Sturdee DW. Treatment of menopausal symptoms: what shall we do now? Lancet 2005;366:409-421

103. Nippita TA, Baber RJ. Premature ovarian failure: a review. Climacteric 2007;10:11-22

104. Sare GM, Gray LJ, Bath PM. Association between hormone replacement therapy and subsequent arterial and venous vascular events: a meta-analysis. Eur Heart J 2008;29:20312041

105. Adomaityte J, Farooq M, Qayyum R. Effect of raloxifene therapy on venous thromboembolism in postmenopausal women. A meta-analysis. Thromb Haemost 2008;99: 338-342

106. Canonico M, Plu-Bureau G, Lowe GD, Scarabin PY. Hormone replacement therapy and risk of venous thromboembolism in postmenopausal women: systematic review and meta-analysis. BMJ 2008;336:1227-1231

107. Samama MM. An epidemiologic study of risk factors for deep vein thrombosis in medical outpatients: the Sirius study. Arch Intern Med 2000;160:3415-3420

108. White HR, Gettner S, Newman J, Trauner KB, Romano SP. Predictors of rehospitalization for symptomatic venous thromboembolismafter total hip arthroplasty. N Eng1 J Med 2000;343:1758-1764

109. Abdollahi M, Cushman M, Rosendaal FR. Obesity: risk of venous thrombosis and the interaction with coagulation factor levels and oral contraceptive use. Thromb Haemost 2003;89:493-498

110. Ageno W, Becattini C, Brighton T, Selby R, Kamphuisen PW. Cardiovascular risk factors and venous thromboembolism: a meta-analysis. Circulation 2008;117:93102

111. Bick RL, Baker WF. Antiphospholipid syndrome and thrombosis. Semin Thromb Hemost 1999;25:333350

112. Wahl DG, Guillemin F, de Maistre E, Perret-Guillaume C, Lecompte T, Thibaut G. Meta-analysis of the risk of venous thrombosis in individuals with antiphospholipid antibodies without underlying autoimmune disease or previous thrombosis. Lupus 1998;7:15-22

113. Galli M, Barbui T. Antiphospholipid syndrome: clinical and diagnostic utility of laboratory tests. Semin Thromb Hemost 2005;31:17-24 
114. Sofi F, Marcucci R, Abbate R, Gensini GF, Prisco D. Lipoprotein (a) and venous thromboembolism in adults: a meta-analysis. Am J Med 2007;120:728-733

115. den Heijer M, Rosendaal FR, Blom HJ, Gerrits WB, Bos GM. Hyperhomocysteinemia and venous thrombosis: a meta-analysis. Thromb Haemost 1998;80:874-877
116. Cattaneo M. Hyperhomocysteinemia and venous thromboembolism. Semin Thromb Hemost 2006;32:716723

117. Simioni P, Sanson BJ, Prandoni P, et al. Incidence of venous thromboembolism in families with inherited thrombophilia. Thromb Haemost 1999;81:198-202 